Received: 27 September 2017

Accepted: 6 February 2018

Published online: 13 March 2018

\section{OPEN Molecular typing of Mycobacterium kansasii using pulsed-field gel electrophoresis and a newly designed variable-number tandem repeat analysis}

Zofia Bakuła ${ }^{1}$, Anna Brzostek², Paulina Borówka ${ }^{3}$, Anna Żaczek ${ }^{4}$, Izabela Szulc-Kiełbik², Agata Podpora ${ }^{1}$, Paweł Parniewski ${ }^{2}$, Dominik Strapagiel $\mathbb{1}^{5}$, Jarosław Dziadek ${ }^{2}$, Małgorzata Proboszcz ${ }^{6}$, Jacek Bielecki ${ }^{1}$, Jakko van Ingen ${ }^{7}$ \& Tomasz Jagielski ${ }^{1}$

Molecular epidemiological studies of Mycobacterium kansasii are hampered by the lack of highlydiscriminatory genotyping modalities. The purpose of this study was to design a new, high-resolution fingerprinting method for $M$. kansasii. Complete genome sequence of the $M$. kansasii ATCC 12478 reference strain was searched for satellite-like repetitive DNA elements comprising tandem repeats. A total of 24 variable-number tandem repeat (VNTR) loci were identified with potential discriminatory capacity. Of these, 17 were used to study polymorphism among $67 \mathrm{M}$. kansasii strains representing six subtypes (I-VI). The results of VNTR typing were compared with those of pulsed-field gel electrophoresis (PFGE) with Asnl digestion. Six VNTRs i.e. (VNTR 1, 2, 8, 14, 20 and 23) allow to differentiate analyzed strains with the same discriminatory capacities as use of a 17-loci panel. VNTR typing and PFGE in conjunction revealed 45 distinct patterns, including 11 clusters with 33 isolates and 34 unique patterns. The Hunter-Gaston's discriminatory index was 0.95 and 0.66 for PFGE and VNTR typing respectively, and 0.97 for the two methods combined. In conclusion, this study delivers a new typing scheme, based on VNTR polymorphism, and recommends it as a first-line test prior to PFGE analysis in a two-step typing strategy for M. kansasii.

Mycobacterium kansasii, a non-tuberculous mycobacterium (NTM), is an opportunistic pathogen with a predilection for causing pulmonary disease. Since only two cases of $M$. kansasii human-to-human transmission have been documented ${ }^{1,2}$, the presumed source of the disease is exposure to environmental reservoirs, particularly municipal tap water ${ }^{3,4}$.

Although M. kansasii is one of the six most frequently isolated NTM species across the world ${ }^{4}$, little is known about the epidemiological aspects of $M$. kansasii infection including its reservoirs, contagiousness, transmission routes, and distribution in different geographical regions and among different human populations. This hampers the implementation of any prophylactic procedures and emergency therapies to control and prevent M. kansasii infections.

An important reason for this scanty knowledge on M. kansasii epidemiology is the lack of powerful tools allowing for intraspecies differentiation or typing. This, in turn, originates from a poor understanding of the genetic composition of $M$. kansasii. Until quite recently $M$. kansasii has been considered an extensively clonal

${ }^{1}$ Department of Applied Microbiology, Institute of Microbiology, Faculty of Biology, University of Warsaw, Warsaw, Poland. ${ }^{2}$ Institute of Medical Biology, Polish Academy of Sciences, Łódź, Poland. ${ }^{3}$ Department of Anthropology, University of Łódź, Łódź, Poland. " Department of Biochemistry and Cell Biology, University of Rzeszów, Rzeszów, Poland. ${ }^{5}$ Biobank Lab, Department of Molecular Biophysics, Faculty of Biology and Environmental Protection, University of Łódź, Łódź, Poland. ${ }^{6}$ Department of Internal Medicine, Pulmonary Diseases and Allergy, Medical University of Warsaw, Warsaw, Poland. ${ }^{7}$ Department of Medical Microbiology, Radboud University Medical Center, Nijmegen, The Netherlands. Correspondence and requests for materials should be addressed to T.J. (email: t.jagielski@biol.uw.edu.pl) 
species $^{5}$. The genetic structure of $M$. kansasii has comprehensively been investigated in two late 1990s studies, which allowed the species to be classified into five distinct subtypes (I-V), based on PCR restriction enzyme analysis (PCR-REA) with the major polymorphic tandem repeat (MPTR) probe, pulsed-field gel electrophoresis (PFGE), amplified fragment length polymorphism (AFLP) analysis, and PCR-REA of the hsp65 gene ${ }^{5,6}$. Somewhat later, two novel types (VI and VII) along with an intermediate I/II and atypical IIB type have been described ${ }^{7,8}$.

Since subtype I represented the majority of $M$. kansasii clinical isolates (42-100\%) in most of the genotyping studies $^{5-20}$, the currently available genotyping schemes do not permit differentiation between those of clinical relevance (disease-related) and those representing merely isolation, without causing a disease. However, as evidenced by Taillard et al., M. kansasii subtype I has particularly high virulence capacities in immunocompetent patients, whereas subtype II is as an opportunistic pathogen infecting immunosuppressed individuals ${ }^{7}$.

A number of molecular markers have been employed to explore the genetic diversity in M. kansasii. Whereas only slight inter-strain polymorphism has been revealed with AFLP analysis, ${ }^{5,10}$, PFGE- and rep-PCR-based methods were more distinctive $e^{5,6,9,13,16,19}$. However, in all those studies, one-fifth (18.6\%) to half of the M. kansasii strains (47\%) were indistinguishable.

Altogether, there is currently no effective genotyping methodology for M. kansasii, sufficient for approaching key issues in the epidemiology of this pathogen. Hence, the formulation of a new technique, with a high discriminatory capacity is strongly required. Molecular typing methods have permitted investigation of the NTM transmission patterns ${ }^{21}$ discrimination between drug resistant and drug susceptible isolates ${ }^{22}$ and identification of isolates with high virulence capacities ${ }^{23}$.

Mycobacterial interspersed repetitive units (MIRUs), a specific class of variable number of tandem repeats (VNTRs), were first described as 46-101-bp DNA elements scattered at 41 loci in the chromosome of $M$. tuberculosis $\mathrm{H} 37 \mathrm{Rv}^{24}$. Variations in the MIRU-VNTR copy number have predisposed these sequences to be used as efficient molecular markers for typing isolates of $M$. tuberculosis complex. Furthermore, since MIRU-VNTR analysis is based on PCR amplification of the respective loci, followed by determination of the sizes of the amplicons by gel electrophoresis, it is considered relatively inexpensive and easy to handle typing method. Over the last 15 years, sequences similar to MIRU-VNTRs in M. tuberculosis, have been identified in several NTM species, including $M$. abscessus, M. avium, M. intracellulare, M. marinum, and M. ulcerans ${ }^{25-30}$, and non-mycobacteria ${ }^{31,32}$ and used for inter-strain differentiation with a varied degree of success.

However, a multi-lous VNTR-based method has never been attempted on M. kansasii.

In this study, a genome of M. kansasii was searched for VNTR-like loci, and based on those found, a new typing scheme for M. kansasii was designed. The method was evaluated on a collection of $67 \mathrm{M}$. kansasii isolates, representatives of six subtypes (I-VI), and compared, in terms of resolution power, performance, and practicality, with PFGE analysis.

\section{Results}

VNTR typing. Among 1,447 VNTRs found in the genome of the M. kansasii ATCC 12478 reference strain (Suppl. Table 4), 24 were selected based on predefined criteria. The characteristics of those loci were described in Suppl. Table 2. Seven loci (VNTR 5, 9, 10, 12, 13, 16, 22) were excluded from the analysis due to lack of amplification (VNTR 9, 13, 22) or multiple bands (VNTR 5, 10, 12, 16, 22), as seen upon electrophoresis.

Different VNTR loci showed different ranges of allelic variability (Table 1). For three loci ${ }^{14,17,24}$ only two allelic variants were detected. Eight loci $i^{3,4,7,11,15,18,21,23}$ were demonstrated as three-allele types, and another two ${ }^{1,6}$ and three ${ }^{8,19,20}$ loci as four-and five-allele types, respectively. The highest level of variation was evidenced for VNTR locus 2, with seven allelic types.

VNTR 19 was the only locus capable of discriminating between all M. kansasii subtypes.

The HGDIs for each VNTR locus are shown in Table 1. The most distinguishing loci were VNTR 8 and VNTR 20 (HGDI = 0.38). Whereas the lowest diversity was observed for VNTR 14 (HGDI =0.09). The schematic representation of allelic diversity at VNTR 8 locus is depicted on Fig. 1.

17-loci VNTR analysis produced 19 distinct profiles in total. There were six clusters with 2-39 isolates per cluster and 13 unique profiles $(\mathrm{GDI}=0.35 ; \mathrm{HGDI}=0.66)$ (Table 1 , Suppl. Figure 2). The clustering rate $(\mathrm{CR})$ of the VNTR method was calculated at $80.6 \%$.

Among 54 M. kansasii subtype I isolates only VNTR 1, 2, 8, and 20 showed variation, generating 11 different VNTR profiles, split into 3 clusters and 8 unique profiles $(\mathrm{GDI}=0.2 ; \mathrm{HGDI}=0.47)$. Cluster "a" contained almost two-thirds of the isolates $(39 / 54 ; 72.2 \%)$, whereas cluster "b" and "c" included five $(5 / 54 ; 9.2 \%)$ and two $(2 / 54$; $3.7 \%)$ isolates, respectively.

Six VNTRs $(1-4,14$, and 23) were polymorphic among $7 \mathrm{M}$. kansasii subtype II isolates, and produced 4 different profiles - a four-isolate $(4 / 7 ; 57.1 \%)$ cluster ("l") and three unique profiles (GDI =0.57, HGDI =0.71).

Profiles "p", "r", "s", and "t" were specific for M. kansasii type III $(n=2)$, IV $(n=1)$ V $(n=2)$, and VI $(n=1)$, respectively.

It is worth noting that the use of only six VNTRs (VNTR 1, 2, 8, 14, 20 and 23) had the same discriminatory power as the use of whole 17-loci panel (GDI $=0.35$; HGDI $=0.66)$.

Of the six VNTR clusters, four (“a", "l”, "p", "s") contained isolates from different countries, two ("a", "p") contained isolates of both environmental and clinical origin, and three ("a", "b", "l") contained isolates of both disease-related and unrelated cases.

Loci, most difficult to assess with respect to the copy number upon gel-sizing (i.e. containing short repeats of $20 \mathrm{bp}$ size or less, namely VNTR 19, 20 and 24) were sequenced to verify estimates of sequence divergence. The copy number of VNTR 19 was in full agreement with that based on gel sizing for the representatives of M. kansasii types I, II and VI. Yet, in the genotype V strain, a single copy difference was noted (Suppl. Table 5). For locus VNTR 20, sequencing results were obtained only for $M$. kansasii type I strain, confirming the 


\begin{tabular}{|c|c|c|c|c|c|c|c|c|c|c|c|c|c|c|c|c|c|c|c|}
\hline \multirow[b]{2}{*}{$\begin{array}{l}\text { VNTR } \\
\text { profile }\end{array}$} & \multicolumn{17}{|c|}{ VNTR locus (HGDI $\left.{ }^{a}\right)$} & \multirow[b]{2}{*}{$\begin{array}{l}\begin{array}{l}\text { No. of } \\
\text { isolates }\end{array} \\
\text { a }\end{array}$} & \multirow{2}{*}{$\begin{array}{l}\text { Total no. } \\
\text { of isolates } \\
\text { (subtype) }\end{array}$} \\
\hline & \begin{tabular}{|l|}
1 \\
$(0.22)$
\end{tabular} & \begin{tabular}{|l|}
2 \\
$(0.32)$
\end{tabular} & \begin{tabular}{|l|}
3 \\
$(0.33)$
\end{tabular} & $\begin{array}{l}4 \\
(0.34)\end{array}$ & \begin{tabular}{|l|}
6 \\
$(0.29)$
\end{tabular} & \begin{tabular}{|l}
7 \\
$(0.33)$
\end{tabular} & \begin{tabular}{|l|}
8 \\
$(0.38)$
\end{tabular} & \begin{tabular}{|l|l}
11 \\
$(0.29)$
\end{tabular} & \begin{tabular}{|l|}
14 \\
$(0.09)$ \\
\end{tabular} & \begin{tabular}{|l|}
15 \\
$(0.14)$
\end{tabular} & \begin{tabular}{|l|}
17 \\
$(0.32)$
\end{tabular} & \begin{tabular}{|l|}
18 \\
$(0.33)$
\end{tabular} & \begin{tabular}{|l|}
19 \\
$(0.34)$
\end{tabular} & \begin{tabular}{|l|}
20 \\
$(0.38)$
\end{tabular} & \begin{tabular}{|l|}
21 \\
$(0.34)$
\end{tabular} & \begin{tabular}{|l|}
23 \\
$(0.2)$
\end{tabular} & \begin{tabular}{|l}
24 \\
$(0.19)$
\end{tabular} & & \\
\hline $\mathrm{a}$ & 13 & 7 & 7 & 4 & 6 & 6 & 5 & 7 & 5 & 6 & 5 & 5 & 11 & 13 & 6 & 3 & 9 & 39 & \multirow{11}{*}{$54(\mathrm{I})$} \\
\hline $\mathrm{b}$ & 13 & 6 & 7 & 4 & 6 & 6 & 5 & 7 & 5 & 6 & 5 & 5 & 11 & 13 & 6 & 3 & 9 & 5 & \\
\hline $\mathrm{c}$ & 13 & 8 & 7 & 4 & 6 & 6 & 5 & 7 & 5 & 6 & 5 & 5 & 11 & 13 & 6 & 3 & 9 & 2 & \\
\hline $\mathrm{d}$ & 13 & 5 & 7 & 4 & 6 & 6 & 5 & 7 & 5 & 6 & 5 & 5 & 11 & 13 & 6 & 3 & 9 & 1 & \\
\hline e & 13 & 7 & 7 & 4 & 6 & 6 & 7 & 7 & 5 & 6 & 5 & 5 & 11 & 13 & 6 & 3 & 9 & 1 & \\
\hline $\mathrm{f}$ & 13 & 8 & 7 & 4 & 6 & 6 & 5 & 7 & 5 & 6 & 5 & 5 & 11 & 12 & 6 & 3 & 9 & 1 & \\
\hline $\mathrm{g}$ & 13 & 7 & 7 & 4 & 6 & 6 & 6 & 7 & 5 & 6 & 5 & 5 & 11 & 13 & 6 & 3 & 9 & 1 & \\
\hline $\mathrm{h}$ & 13 & 7 & 7 & 4 & 6 & 6 & 3 & 7 & 5 & 6 & 5 & 5 & 11 & 13 & 6 & 3 & 9 & 1 & \\
\hline i & 13 & 7 & 7 & 4 & 6 & 6 & 5 & 7 & 5 & 6 & 5 & 5 & 11 & 12 & 6 & 3 & 9 & 1 & \\
\hline j & 13 & 4 & 7 & 4 & 6 & 6 & 5 & 7 & 5 & 6 & 5 & 5 & 11 & 13 & 6 & 3 & 9 & 1 & \\
\hline $\mathrm{k}$ & 12 & 7 & 7 & 4 & 6 & 6 & 5 & 7 & 5 & 6 & 5 & 5 & 11 & 13 & 6 & 3 & 9 & 1 & \\
\hline 1 & 13 & 7 & 2 & 3 & 1 & 0 & 3 & 2 & 5 & 6 & 1 & 0 & 12 & 12 & 4 & 3 & 3 & 4 & \multirow{4}{*}{$7(\mathrm{II})$} \\
\hline $\mathrm{m}$ & 13 & 7 & 2 & 3 & 1 & 0 & 3 & 2 & $\mathrm{NP}^{\mathrm{b}}$ & 6 & 1 & 0 & 12 & 12 & 4 & 3 & 3 & 1 & \\
\hline $\mathrm{n}$ & 13 & 7 & 2 & 3 & 1 & 0 & 3 & 2 & NP & 6 & 1 & 0 & 12 & 12 & 4 & 0 & 3 & 1 & \\
\hline o & 0 & 2 & 4 & \begin{tabular}{|l|}
0 \\
\end{tabular} & 1 & 0 & 3 & 2 & 4 & 6 & 1 & 0 & 12 & 12 & 4 & 3 & 3 & 1 & \\
\hline $\mathrm{p}$ & 0 & 7 & 2 & 0 & 4 & 1 & NP & 7 & 5 & MPc & 1 & 1 & NP & 8 & NP & 5 & 9 & 2 & 2 (III) \\
\hline $\mathrm{r}$ & MP & 3 & 2 & 0 & 1 & 0 & 0 & 1 & 5 & 6 & NP & 0 & 6 & NP & NP & 0 & 9 & 1 & 1 (IV) \\
\hline $\mathrm{s}$ & 14 & 7 & 2 & 0 & 6 & 0 & 3 & 2 & 5 & 14 & 1 & 0 & 7 & 2 & NP & MP & 9 & 2 & $2(\mathrm{~V})$ \\
\hline$t$ & 12 & 7 & MP & 0 & 5 & NP & 5 & 1 & 5 & 0 & 1 & 0 & 10 & 0 & 12 & 0 & 9 & 1 & $1(\mathrm{VI})$ \\
\hline
\end{tabular}

Table 1. VNTR typing profiles of 67 M. kansasii isolates. Bold font indicates VNTR locus at which allelic variability within the same subtype was observed; ${ }^{a}$ The Hunter-Gaston's discriminatory index; ${ }^{b} \mathrm{NP}$, no PCR product; ${ }^{\mathrm{c}} \mathrm{MP}$, multiple-band profile.

number of copies calculated from the gel analysis of PCR products. Sequence analysis confirmed also the electrophoretically-deduced copy number at locus VNTR 24 for M. kansasii types I, II, and IV and VI.

PFGE. Three restriction endonucleases, that is AseI, DraI, and XbaI were attempted in PFGE profiling. Whereas, the latter two consistently resulted in DNA band smearing, thus making the profiles unreadable (data not shown), the use of AseI provided good-quality restriction patterns with clear band separation. PFGE with AseI was performed on the entire study sample (67 isolates).

PFGE produced 33 distinct patterns, each consisting of 11-24 bands with sizes ranging between 48.5 to $485 \mathrm{~kb}$. The patterns were separated into 12 clusters, each containing 2-9 isolates, and 21 unique patterns $(\mathrm{GDI}=0.49$; HGDI $=0.95)$ (Table 2, Suppl. Figure 1).

The CR of the PFGE analysis was calculated at $68.6 \%$.

Fifty-four M. kansasii subtype I isolates formed 25 different profiles split into 8 clusters and 17 unique patterns $(\mathrm{GDI}=0.46$; $\mathrm{HGDI}=0.93)$. Of the clusters, the most abundant were $\mathrm{F}$ and $\mathrm{H}$, each comprising 9 isolates $(9 / 54$; $16.7 \%)$, followed by $\mathrm{R}$ and $\mathrm{U}$, of four isolates, each $(4 / 54 ; 7.4 \%), \mathrm{M}, \mathrm{S}$, and $\mathrm{X}$, each with three isolates $(3 / 54 ; 5.6 \%)$, and D with two isolates $(2 / 54 ; 3.7 \%)$.

Among seven M. kansasii type II isolates, there were two clusters (AC, AE), with two isolates, each (2/7; $28.6 \%)$, and three unique profiles (GDI $=0.71 ; \mathrm{HGDI}=0.9)$.

Type III $(n=2)$ and IV $(n=1)$ isolates shared identical PFGE pattern $(\mathrm{AI})$, whereas type V $(n=2)$ and VI $(n=1)$ isolates had type-specific profiles, that is $\mathrm{AB}$ and $\mathrm{L}$, respectively. Although some clusters were homogenous with respect to isolate's geographical origin (e.g. F, R, U, AC, AE), source of recovery (clinical/environmental) (e.g. $\mathrm{F}, \mathrm{H}, \mathrm{R}, \mathrm{U}, \mathrm{AB}$ ), and clinical relevance (e.g. S, AC, AE), clusters allocating isolates of different pathogenic status (F, $\mathrm{H}, \mathrm{R}, \mathrm{U}, \mathrm{X})$, from different countries $(\mathrm{H}, \mathrm{M}, \mathrm{AI}, \mathrm{AB})$ or sources $(\mathrm{M}, \mathrm{AI})$ were also observed.

Combined analysis. Four ("a", "b", "c", "l") of the six VNTR clusters were subdivided by PFGE analysis (Table 3; Fig. 2). Within a major VNTR cluster "a", comprising 39 isolates, 23 distinct pulsotypes (A-J, M-Z, AG, AH) could be distinguished. PFGE also differentiated within VNTR clusters "b" (5 isolates), "l" (4 isolates), and "c" (2 isolates), creating three (U, X, AA), two (AC, AE), and two (F, M) pulsotypes, respectively.

Contrariwise, of 12 PFGE clusters, nine (D, F, H, M, R, S, U, X, AI) were further separated upon VNTR typing (Table 3). Two major pulsotype clusters ( $F$ and H), of nine isolates each, could be subdivided into four ("a", "c", "e", "f") and two ("a", "h") VNTR clusters, respectively. Clusters R and U, each comprising four isolates, were both split into two VNTR clusters ("a", "j" and "a", "b"). A three-isolate cluster M and a two-isolate cluster D were split into three ("a", "c", "k"), and two ("a", "d") VNTR clusters, accordingly. Patterns S, X, and AI, each with three isolates, were subdivided into two different VNTR clusters (“a”, "i”; "m”, "b”; “p”, "r").

A combined VNTR-PFGE analysis resolved 45 distinct patterns, separated into 11 clusters totaling 33 isolates and 34 unique patterns $(\mathrm{GDI}=0.67 ; \mathrm{HGDI}=0.97)$. The $\mathrm{CR}$ of the combined analysis was $49.2 \%$. 
A

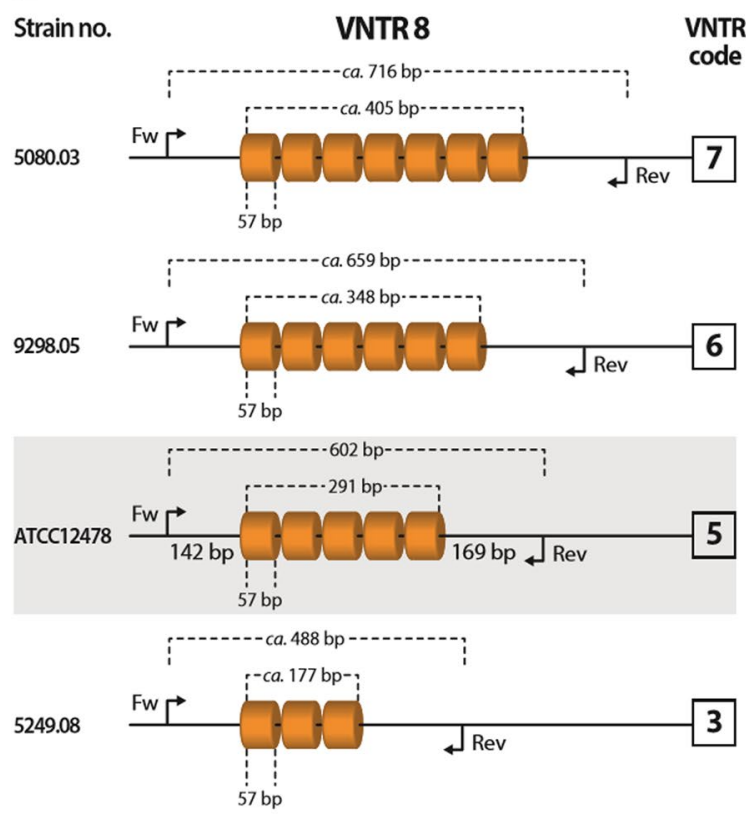

\section{B}

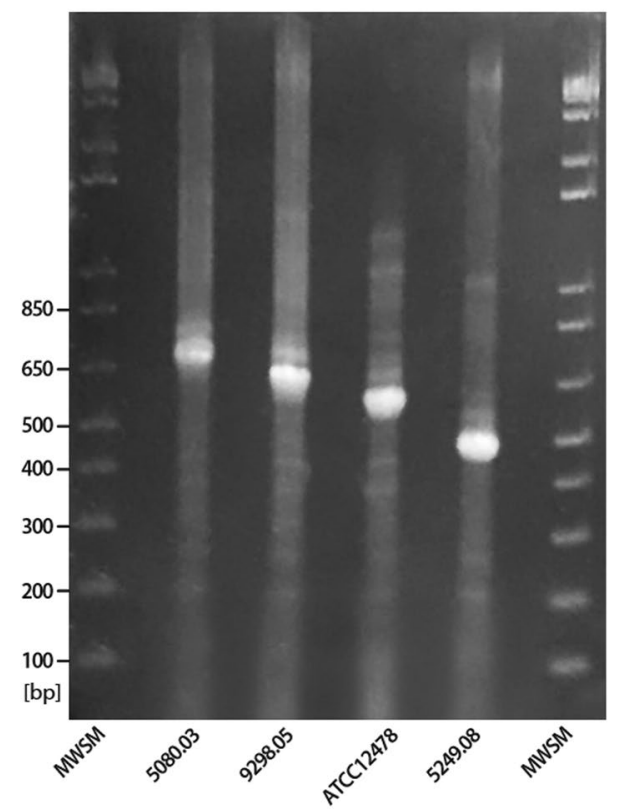

Figure 1. Polymorphism of the VNTR 8 locus. (A) Schematic representation of allelic diversity of the VNTR 8 locus among isolates under the study. (B) PCR-amplified VNTR 8 locus in four M. kansasii isolates showing bands of different length corresponding to different number of repeats within the locus. MWSM, molecularweight size marker (100 bp DNA ladder, New England BioLabs, Ipswich, USA).

M. kansasii subtype I isolates were divided into 36 different profiles or seven clusters, 2-8 isolates, each and 29 unique profiles $(\mathrm{GDI}=0.67 ; \mathrm{HGDI}=0.96)$. The largest clusters $(\mathrm{H} / \mathrm{a} ; \mathrm{F} / \mathrm{a})$ comprised eight $(8 / 67 ; 11.9 \%)$ and six isolates $(6 / 67 ; 8.9 \%)$, respectively.

Subtype II isolates either belonged to one of two clusters (1/AC; 1/AE), each of two isolates or harbored unique profiles $(\mathrm{GDI}=0.71 ; \mathrm{HGDI}=0.9)$.

Isolates of subtype III $(n=2)$, IV $(n=1), \mathrm{V}(n=1)$, and VI $(n=1)$ had their type-specific merged profiles.

Of the eleven VNTR-PFGE clusters, three (a/H; p/AI; s/AB) contained isolates from more than one country and three $(\mathrm{a} / \mathrm{F} ; \mathrm{a} / \mathrm{H} ; \mathrm{b} / \mathrm{X})$ contained both clinically relevant and irrelevant isolates. One cluster (p/AI) accommodated isolates of both clinical and environmental origin.

\section{Discussion}

In the epidemiology of infectious diseases, including those of mycobacterial etiology, the key issue is disclosing sources of infection, transmission links, and dissemination in the environment. For this to be accomplished, high resolution inter-strain discrimination or typing is of utmost importance ${ }^{33}$.

Molecular typing methods have substantially improved our knowledge of the epidemiology of mycobacterial infections and genuinely assisted in the fight against them.

A significant source of the genetic polymorphism in mycobacteria, and one of the main driving forces of their genome evolution, are repetitive DNA elements. One such large group of repeated DNA are insertion sequences (ISs) ${ }^{5,33-35}$. Equally important, as ISs, group of repetitive DNA elements are tandem repeats (TRs) alias variable number of tandem repeats (VNTRs), which are short direct repeats organized as head-to-tail arrays, classified as micro-, mini- or macrosatellites, depending on their repeat unit size ${ }^{36}$. The first VNTRs described in mycobacteria were those identified in $M$. tuberculosis, collectively referred to as mycobacterial interspersed repetitive units (MIRUs) ${ }^{37}$. Although the panel of MIRU-VNTR loci has been modified over the years, the standardized 24-locus MIRU-VNTR typing is currently recognized as the reference typing system for $M$. tuberculosis complex ${ }^{38-40}$. The principle of the VNTR-based typing modalities involves PCR amplification of a predefined set of VNTR loci, by using primers annealing to their flanking sequences, and amplicon size determination to deduce the number of TR units within each locus. At the end, a numerical code is developed, which corresponds to the numbers of repeats at each locus and serves as a strain's molecular fingerprint. All this renders multiple-locus VNTR analysis (MLVA) robust, easy-to-perform, time- and cost-effective. The portability of the results (MLVA codes) facilitates their storage and exchange between laboratories. More importantly, the discriminatory power of MLVA is often high, surpassing that of other typing schemes ${ }^{41,42}$.

VNTRs have also been described in several NTM species, including M. abscessus, M. avium, M. intracellulare, and M. marinum ${ }^{25,27,29,43-46}$. However, until this study, the only evidence for the existence of VNTR-like loci in $M$. kansasii came from a 30-year-old study by Hermans et al. ${ }^{47}$. DNA homologous to a major polymorphic tandem repeat (MPTR), originating from $M$. tuberculosis, was shown to be present in M. kansasii and M. gordonae ${ }^{47}$. In this work, the VNTRs were searched directly in the M. kansasii genomes. Based on the applied criteria, a total of 24 VNTR loci were identified in the genome of the M. kansasii ATCC 12478 reference strain. Only 17 of the 


\begin{tabular}{|c|c|c|}
\hline PFGE profile & No. of isolates & $\begin{array}{l}\text { Total no. of isolates } \\
\text { (subtype) }\end{array}$ \\
\hline A & 1 & \multirow{25}{*}{$54(\mathrm{I})$} \\
\hline $\mathrm{B}$ & 1 & \\
\hline $\mathrm{C}$ & 1 & \\
\hline $\mathrm{D}$ & 2 & \\
\hline $\mathrm{E}$ & 1 & \\
\hline $\mathrm{F}$ & 9 & \\
\hline G & 1 & \\
\hline $\mathrm{H}$ & 9 & \\
\hline I & 1 & \\
\hline $\mathrm{J}$ & 1 & \\
\hline $\mathrm{K}$ & 1 & \\
\hline $\mathrm{M}$ & 3 & \\
\hline $\mathrm{N}$ & 1 & \\
\hline $\mathrm{O}$ & 1 & \\
\hline $\mathrm{P}$ & 1 & \\
\hline $\mathrm{R}$ & 4 & \\
\hline S & 3 & \\
\hline $\mathrm{T}$ & 1 & \\
\hline $\mathrm{U}$ & 4 & \\
\hline $\mathrm{W}$ & 1 & \\
\hline $\mathrm{X}$ & 3 & \\
\hline $\mathrm{Z}$ & 1 & \\
\hline AA & 1 & \\
\hline AG & 1 & \\
\hline $\mathrm{AH}$ & 1 & \\
\hline $\mathrm{Y}$ & 1 & \multirow{5}{*}{7 (II) } \\
\hline $\mathrm{AC}$ & 2 & \\
\hline $\mathrm{AD}$ & 1 & \\
\hline $\mathrm{AE}$ & 2 & \\
\hline $\mathrm{AF}$ & 1 & \\
\hline \multirow{2}{*}{ AI } & 2 & 2 (III) \\
\hline & 1 & 1 (IV) \\
\hline $\mathrm{AB}$ & 2 & $2(\mathrm{~V})$ \\
\hline $\mathrm{L}$ & 1 & $1(\mathrm{~V})$ \\
\hline
\end{tabular}

Table 2. PFGE profiles of 67 M. kansasii isolates.

VNTR loci were applicable as genetic markers in this species fingerprinting. Furthermore, the use of only six VNTRs (VNTR 1, 2, 8, 14, 20 and 23) showed identical strain polymorphism as the use of a whole 17-loci panel. The advantage of using this smaller panel of VNTRs is a reduction in time and cost of the analysis by almost three-fold.

VNTR locus 19 was efficient in differentiating between all $M$. kansasii types except type III, whose isolates failed to produce a PCR product. Nevertheless, given the rarity of type III, PCR amplification of VNTR locus 19 offers an alternative to more lengthy, standard PCR-REA assays, for discrimination of M. kansasii genotypes, including two most prevalent, clinical types I and II.

The reason for the detected difference in the VNTR 19 copy number for $M$. kansasii type V deduced from gel sizing (i.e. 8 copies) and direct sequencing (i.e. 7 copies) may lie in the miscalculation of the band size on the gel. Inaccurate allele calling is one of the major nontechnical error affecting the reproducibility of the VNTR typing ${ }^{48}$.

The overall discriminatory power of the 17-locus typing assay was calculated at 0.66 . This was somewhat lower than what was reported for VNTR typing schemes in other NTM, including M. abscessus (13-18 loci, HGDI $=0.96-0.98)^{29,49,50}$, M. avium (5-8 loci, HGDI from 0.74 to 0.95$)^{44,51-53}$ or M. intracellulare (15-16 loci, $0.98-0.99)^{27,45,54,55}$. At the same time, the genetic diversity of the population studied, measured by the number of different VNTR profiles identified per study sample, was found to be rather moderate when compared to other VNTR-typed NTM (GDI $=0.35$ vs $0.1-0.84)^{25,27,29,44,54-56}$.

As far as the evolutionary genomics is concerned, mycobacteria are remarkably homogeneous at the molecular level. This is also reflected by a slow molecular clock of VNTR loci. For instance, in M. tuberculosis, a mean mutation rate per VNTR locus per year was estimated at $10^{-4}$ which translates in a mean of 0.05 pairwise (single-locus) changes per 24-locus genotype expected in sets of isolates originated from a same clone over 10 years ${ }^{57}$. The low discriminatory power of the 17-locus set for M. kansasii compared to other NTM may indicate that the rate of VNTR variation in M. kansasii is exceptionally low. 


\begin{tabular}{|c|c|c|c|c|c|c|c|c|c|c|c|c|c|c|c|c|c|c|c|c|}
\hline \multirow[b]{2}{*}{$\begin{array}{l}\text { PFGE } \\
\text { profile }\end{array}$} & \multirow[b]{2}{*}{$\begin{array}{l}\text { VNTR } \\
\text { profile }\end{array}$} & \multicolumn{17}{|c|}{ VNTR locus } & \multirow[b]{2}{*}{$\begin{array}{l}\text { No. of } \\
\text { isolates }\end{array}$} & \multirow{2}{*}{\begin{tabular}{|l|} 
Total no. \\
of isolates \\
(subtype)
\end{tabular}} \\
\hline & & 1 & 2 & 3 & 4 & 6 & 7 & 8 & 11 & 14 & 15 & 17 & 18 & 19 & 20 & 21 & 23 & 24 & & \\
\hline A & \multirow{3}{*}{ a } & \multirow{3}{*}{13} & \multirow{3}{*}{7} & \multirow{3}{*}{7} & \multirow{3}{*}{4} & \multirow{3}{*}{6} & \multirow{3}{*}{6} & \multirow{3}{*}{5} & \multirow{3}{*}{7} & \multirow{3}{*}{5} & & & & & & & & & 1 & \\
\hline B & & & & & & & & & & & 6 & 5 & 5 & 11 & 13 & 6 & 3 & 9 & 1 & \\
\hline $\mathrm{C}$ & & & & & & & & & & & & & & & & & & & 1 & \\
\hline & $\mathrm{a}$ & 13 & 7 & 7 & 4 & 6 & 6 & 5 & 7 & 5 & 6 & 5 & 5 & 11 & 13 & 6 & 3 & 9 & 1 & \\
\hline D & d & 13 & 5 & 7 & 4 & 6 & 6 & 5 & 7 & 5 & 6 & 5 & 5 & 11 & 13 & 6 & 3 & 9 & 1 & \\
\hline $\mathrm{E}$ & $\mathrm{a}$ & 13 & 7 & 7 & 4 & 6 & 6 & 5 & 7 & 5 & 6 & 5 & 5 & 11 & 13 & 6 & 3 & 9 & 1 & \\
\hline & $\mathrm{a}$ & 13 & 7 & 7 & 4 & 6 & 6 & 5 & 7 & 5 & 6 & 5 & 5 & 11 & 13 & 6 & 3 & 9 & 6 & \\
\hline$F$ & c & 13 & 8 & 7 & 4 & 6 & 6 & 5 & 7 & 5 & 6 & 5 & 5 & 11 & 13 & 6 & 3 & 9 & 1 & \\
\hline $\mathrm{F}$ & e & 13 & 7 & 7 & 4 & 6 & 6 & 7 & 7 & 5 & 6 & 5 & 5 & 11 & 13 & 6 & 3 & 9 & 1 & \\
\hline & f & 13 & 8 & 7 & 4 & 6 & 6 & 5 & 7 & 5 & 6 & 5 & 5 & 11 & 12 & 6 & 3 & 9 & 1 & \\
\hline G & $\mathrm{a}$ & 13 & 7 & 7 & 4 & 6 & 6 & 5 & 7 & 5 & 6 & 5 & 5 & 11 & 13 & 6 & 3 & 9 & 1 & \\
\hline $\mathrm{H}$ & $\mathrm{a}$ & 13 & 7 & 7 & 4 & 6 & 6 & 5 & 7 & 5 & 6 & 5 & 5 & 11 & 13 & 6 & 3 & 9 & 8 & \\
\hline H & $\mathrm{h}$ & 13 & 7 & 7 & 4 & 6 & 6 & 3 & 7 & 5 & 6 & 5 & 5 & 11 & 13 & 6 & 3 & 9 & 1 & \\
\hline I & , & 13 & 7 & 7 & 4 & 6 & 6 & 5 & 7 & 5 & 6 & 5 & 5 & 11 & 13 & 6 & 3 & o & 1 & \\
\hline $\mathrm{J}$ & a & 13 & 7 & 7 & 4 & 6 & 6 & 5 & 7 & 5 & 6 & 5 & 5 & 11 & 13 & 6 & 3 & 9 & 1 & \\
\hline $\mathrm{K}$ & $\mathrm{g}$ & 13 & 7 & 7 & 4 & 6 & 6 & 6 & 7 & 5 & 6 & 5 & 5 & 11 & 13 & 6 & 3 & 9 & 1 & \\
\hline & $\mathrm{a}$ & \begin{tabular}{|l|}
13 \\
\end{tabular} & 7 & 7 & 4 & 6 & 6 & 5 & 7 & 5 & 6 & 5 & 5 & 11 & 13 & 6 & 3 & 9 & 1 & \\
\hline $\mathrm{M}$ & c & 13 & 8 & 7 & 4 & 6 & 6 & 5 & 7 & 5 & 6 & 5 & 5 & 11 & 13 & 6 & 3 & 9 & 1 & \\
\hline & $\mathrm{k}$ & 12 & 7 & 7 & 4 & 6 & 6 & 5 & 7 & 5 & 6 & 5 & 5 & 11 & 13 & 6 & 3 & 9 & 1 & $54(1)$ \\
\hline $\mathrm{N}$ & & & & & & & & & & & & & & & & & & & 1 & \\
\hline $\mathrm{O}$ & $\mathrm{a}$ & 13 & 7 & 7 & 4 & 6 & 6 & 5 & 7 & 5 & 6 & 5 & 5 & 11 & 13 & 6 & 3 & 9 & 1 & \\
\hline $\mathrm{P}$ & & & & & & & & & & & & & & & & & & & 1 & \\
\hline$P$ & $\mathrm{a}$ & 13 & 7 & 7 & 4 & 6 & 6 & 5 & 7 & 5 & 6 & 5 & 5 & 11 & 13 & 6 & 3 & 9 & 3 & \\
\hline R & j & 13 & 4 & 7 & 4 & 6 & 6 & 5 & 7 & 5 & 6 & 5 & 5 & 11 & 13 & 6 & 3 & 9 & 1 & \\
\hline $\mathrm{s}$ & $\mathrm{a}$ & 13 & 7 & 7 & 4 & 6 & 6 & 5 & 7 & 5 & 6 & 5 & 5 & 11 & 13 & 6 & 3 & 9 & 2 & \\
\hline S & i & 13 & 7 & 7 & 4 & 6 & 6 & 5 & 7 & 5 & 6 & 5 & 5 & 11 & 12 & 6 & 3 & 9 & 1 & \\
\hline $\mathrm{T}$ & $\mathrm{a}$ & \begin{tabular}{|l|}
13 \\
\end{tabular} & 7 & 7 & 4 & 6 & 6 & 5 & 7 & 5 & 6 & 5 & 5 & 11 & \begin{tabular}{|l|}
13 \\
\end{tabular} & 6 & 3 & 9 & 1 & \\
\hline Ut & $\mathrm{a}$ & 13 & 7 & 7 & 4 & 6 & 6 & 5 & 7 & 5 & 6 & 5 & 5 & 11 & 13 & 6 & 3 & 9 & 2 & \\
\hline U & $\mathrm{b}$ & 13 & 6 & 7 & 4 & 6 & 6 & 5 & 7 & 5 & 6 & 5 & 5 & 11 & 13 & 6 & 3 & 9 & 2 & \\
\hline $\mathrm{W}$ & $\mathrm{a}$ & 13 & 7 & 7 & 4 & 6 & 6 & 5 & 7 & 5 & 6 & 5 & 5 & 11 & 13 & 6 & 3 & 9 & 1 & \\
\hline $\mathrm{x}$ & $\mathrm{a}$ & 13 & 7 & 7 & 4 & 6 & 6 & 5 & 7 & 5 & 6 & 5 & 5 & 11 & 13 & 6 & 3 & 9 & 1 & \\
\hline$X$ & $\mathrm{~b}$ & \begin{tabular}{|l|}
13 \\
\end{tabular} & 6 & 7 & 4 & 6 & 6 & 5 & 7 & 5 & 6 & 5 & 5 & 11 & \begin{tabular}{|l|}
13 \\
\end{tabular} & 6 & 3 & 9 & 2 & \\
\hline $\mathrm{Z}$ & $\mathrm{a}$ & \begin{tabular}{|l|}
13 \\
\end{tabular} & 7 & 7 & 4 & 6 & 6 & 5 & 7 & 5 & 6 & 5 & 5 & 11 & 13 & 6 & 3 & 9 & 1 & \\
\hline $\mathrm{AA}$ & $\mathrm{b}$ & 13 & 6 & 7 & 4 & 6 & 6 & 5 & 7 & 5 & 6 & 5 & 5 & 11 & 13 & 6 & 3 & 9 & 1 & \\
\hline AG & , & 13 & 7 & 7 & 4 & ( & ( & 5 & 7 & 5 & 6 & 5 & & & 13 & 6 & 3 & 9 & 1 & \\
\hline $\mathrm{AH}$ & a & 13 & 7 & 7 & 4 & 6 & 6 & 5 & 7 & 5 & 6 & 5 & 5 & 11 & 13 & 6 & 3 & 9 & 1 & \\
\hline $\mathrm{Y}$ & $\mathrm{m}$ & 13 & 7 & 2 & 3 & 1 & 0 & 3 & 2 & $\mathrm{NP}^{\mathbf{a}}$ & 6 & 1 & 0 & 12 & 12 & 4 & 3 & 3 & 1 & \\
\hline $\mathrm{AC}$ & 1 & 13 & 7 & 2 & 3 & 1 & 0 & 3 & 2 & 5 & 6 & 1 & 0 & 12 & 12 & 4 & 3 & 3 & 2 & \\
\hline $\mathrm{AD}$ & $\mathrm{n}$ & 13 & 7 & 2 & 3 & 1 & 0 & 3 & 2 & NP & 6 & 1 & 0 & 12 & 12 & 4 & 0 & 3 & 1 & 7 (II) \\
\hline $\mathrm{AE}$ & 1 & 13 & 7 & 2 & 3 & 1 & 0 & 3 & 2 & 5 & 6 & 1 & 0 & 12 & 12 & 4 & 3 & 3 & 2 & \\
\hline $\mathrm{AF}$ & o & 0 & 2 & 4 & 0 & 1 & 0 & 3 & 2 & 4 & 6 & 1 & 0 & 12 & 12 & 4 & 3 & 3 & 1 & \\
\hline $\mathrm{AI}$ & $\mathrm{p}$ & 0 & 7 & 2 & 0 & 4 & 1 & NP & 7 & 5 & MP & 1 & 1 & NP & 8 & NP & 5 & 9 & 2 & 2 (III) \\
\hline Al & $\mathrm{r}$ & $\mathrm{MP}^{\mathrm{b}}$ & 3 & 2 & 0 & 1 & 0 & 0 & 1 & 5 & 6 & NP & 0 & 6 & NP & NP & 0 & 9 & 1 & 1 (IV) \\
\hline $\mathrm{AB}$ & $\mathrm{s}$ & 14 & 7 & 2 & 0 & 6 & 0 & 3 & 2 & 5 & 14 & 1 & 0 & 7 & 2 & NP & MP & 9 & 2 & $2(\mathrm{~V})$ \\
\hline $\mathrm{L}$ & $t$ & 12 & 7 & MP & 0 & 5 & $\mathrm{NP}^{\mathrm{b}}$ & 5 & 1 & 5 & 0 & 1 & 0 & 10 & 0 & 12 & 0 & 9 & 1 & $1(\mathrm{VI})$ \\
\hline
\end{tabular}

Table 3. Combined analysis of VNTR and PFGE profiling results of $67 \mathrm{M}$. kansasii isolates. Bold font indicates VNTR locus at which allelic variability within the same subtype was observed; ${ }^{\mathrm{N} P}$, no PCR product; ${ }^{\mathrm{M}} \mathrm{MP}$, multiple-band profile.

The clustering rate, often used as a proxy for transmission or an outbreak, exceeded, with our method, $80 \%$. This was higher than the analogous rate for M. abscessus $(34.2-48.2 \%)^{29,49}$ or M. intracellulare $(31-55 \%)^{27,54,56}$ and lower than for M. avium (95.6-95.9\% $)^{25,44}$. Given a long collection time of the study sample (16 years) and the fact that the isolates were of different origins (both environmental and clinical), and from different geographical locales, the overall high CR reflects genome homoplasy, which is a product of convergent evolution, rather than ongoing active transmission. 

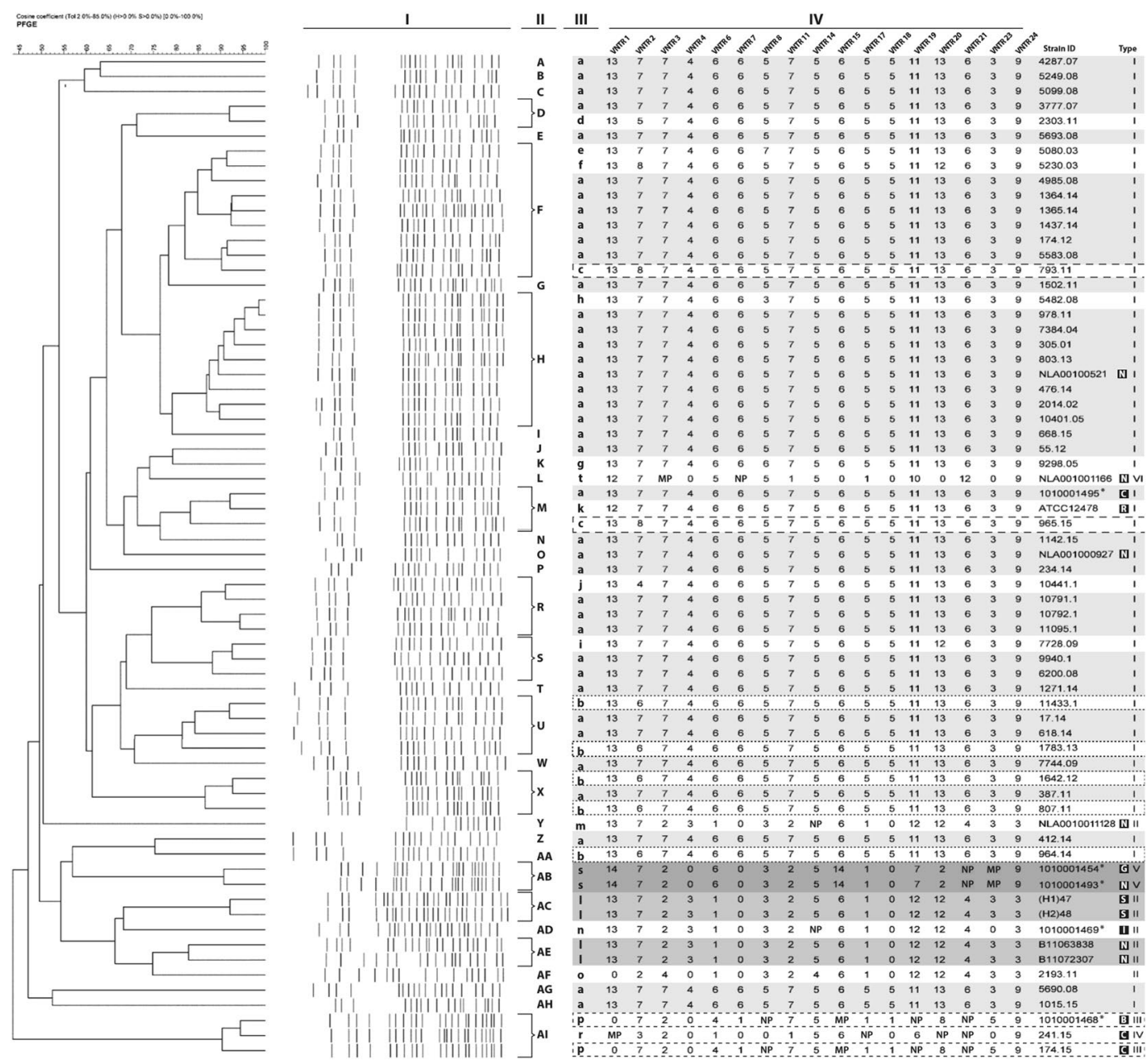

Figure 2. PFGE and VNTR analysis of analyzed isolates. A dendrogram constructed for $67 \mathrm{M}$. kansasii isolates, based on the joint results of 17-loci VNTR and PFGE profiling. I- PFGE banding patterns; II, III- Designation of PFGE (II) and VNTR profiles; IV- TR copy number at each of the 17 loci (VNTR code); VNTR codes highlighted with an identical shade of grey or boxed by dashed lines of different dash spacing correspond to different VNTR clusters; *Strains of environmental origin; Letters in black squares refer to country of strain isolation; N- the Netherlands; C- the Czech Republic; R- reference strain; G- Germany; S- Spain; I- Italy; BBelgium; NP, no PCR product; MP, multiple-band profile. Due to large number of analysed isolates, the samples were derived from multiple experiments. The gels were processed using BioNumerics ver. 5.0 software (Applied Maths, Sint-Martens-Latem, Belgium) software in parallel.

Interestingly, isolate 2193.11 had VNTR pattern somewhat different than other subtype II isolates in loci 1, 2, 3 and 4. Its unique VNTR profile might be associated with geographical origin (this isolate was the only subtype II isolate from Poland).

Since the mid-1990s, PFGE has been the mainstay for molecular typing of NTM. Although, a number of methods have been introduced, over these two decades, PFGE still holds the leading position as a typing scheme in the molecular epidemiological studies of NTM diseases, including those due to M. kansasii ${ }^{33,58}$. This is because PFGE outrivals most of the later-invented typing methods in terms of discriminatory potential ${ }^{5,59}$.

The results from this study shows PFGE comparably discriminatory as in a study by Zhang et al. ${ }^{13}$. By performing three PFGE assays, with different enzymes (XbaI, DraI, and AseI) the GDI was similar between the assays and much the same as in our analysis $(0.39-0.49 \text { vs } 0.49)^{13}$. Much lower GDI values for M. kansasii PFGE typing were reported by other authors, even when using the same enzymes, AseI (GDI $=0.25)$ or DraI $(0.04-0.23)$ and SpeI $(\mathrm{GDI}=0.18)^{5-7,19}$.

As for the clustering, our results were again close to those of Zhang et al. ${ }^{13}$ (CR $=68 \%$ vs $\left.61-69 \%\right)$, albeit rather distant to those from the remaining four aforesaid studies, with the clustering rate ranging from 88.3 to $99.3 \%{ }^{5-7,19}$. These differences may be explained by geographical- and/or population-related specificities of the study samples. Also, some technicalities might be at play. Since PFGE depends chiefly on DNA quality, the typing results can be influenced by a method of DNA isolation, electrophoresis/running conditions, and the puslotype interpretative criteria applied (e.g. correlation algorithms, cutoff values). Whereas PFGE is the most powerful typing system for M. kansasii, it is time-consuming, labor-intensive, and resource- and expertise-demanding. Thus, a new, simple, cost-effective and high-throughput typing method would be of great advantage. All these criteria 
are met by VNTR typing, designed in this study. Yet, the discriminatory ability of MLVA was lower compared to PFGE analysis. This was apparent both from the GDI (0.35 vs 0.49) and HGDI (0.66 vs 0.95$)$ scores.

Encouragingly, when the two methods were used together, the resolution power of such combination increased over that of PFGE alone, as reflected by both diversity and discriminatory indexes (GDI $=0.49$ vs 0.67 ; HGDI $=0.95$ vs 0.97 ). At the same time, the clustering rate noticeably decreased by $31 \%$ and $19 \%$ when compared with MLVA and PFGE alone, respectively ( $49 \%$ vs $68 \%$ and $80 \%$ ). These observations prompted us to propose a two-step typing strategy for M. kansasii, which involves MLVA as a first screening method, performed on the entire study sample, followed by PFGE profiling, performed only within the VNTR-defined clusters.

In conclusion, this study delivers a new typing scheme, based on VNTR polymorphisms, and recommends it as a first-line test prior to PFGE analysis in a two-step typing strategy for $M$. kansasii. This strategy, though requiring evaluation against large-scale samples, offers a promising tool for mapping outbreaks and delineating transmission patterns of $M$. kansasii infections.

\section{Methods}

Isolates. A total of $67 \mathrm{M}$. kansasii isolates, representing six of the species subtypes (I-VI) were included in the study (Suppl. Table 1). The isolates were purchased from the American Type Culture Collection (ATCC) $(n=1)$ or collected over a period of 2000-2015 from Poland $(n=51)$, the Netherlands $(n=7)$, the Czech Republic $(n=3)$, Spain $(n=2)$, Belgium $(n=1)$, Germany $(n=1)$ and Italy $(n=1)$. Sixty-two isolates were of clinical origin and represented as many unrelated patients diagnosed as having (or not) M. kansasii disease, according to the criteria of the American Thoracic Society (ATS) ${ }^{3}$. Five isolates were recovered from different environmental sites (Suppl. Table 1).

The isolates were identified as M. kansasii by using high pressure liquid chromatography (HPLC) methodology, in accordance with the Centers for Disease Control and Prevention (CDC) guidelines ${ }^{60}$ or by means of the GenoType Mycobacterium CM/AS assay (Hain Lifescience, Nehren, Germany).

All isolates were cultured on Löwenstein-Jensen (L-J) medium. Genomic DNA was extracted with the AMPLICOR Respiratory Specimen Preparation Kit (Roche, Basel, Switzerland). Subtyping of M. kansasii isolates was performed upon PCR-REA analysis for the $h s p 65, r p o B$, and $t u f$ genes, as previously described ${ }^{61-63}$.

PFGE. The PFGE analysis was performed as described previously by Kwenda et al. ${ }^{19}$ with modifications (Suppl. Materials and Methods).

The gel images were analyzed using BioNumerics ver. 5.0 software (Applied Maths, Sint-Martens-Latem, Belgium). Three molecular-weight size marker (MWSM) lanes in each 15-well gel enabled normalization within and across gels.

Cosine correlation algorithm was used to define PFGE profiles. Band positions were assigned manually, with computer assistance, and the band tolerance was set at $2 \%$. Two isolates exhibiting $>80.4 \%$ profile similarity were considered clonal. This cut-off value was derived empirically from an analysis of a number of PFGE profiles of the same isolate, in two independent PFGE assays.

Search for VNTR loci and VNTR typing. The whole-genome sequence of the ATCC 12478 M. kansasii reference strain (GenBank, NCBI, Reference Sequence: NC_022663.1) was screened for repetitive DNA elements with the Tandem Repeats Finder Version $4.00^{64}$ and visualized by the Vector NTI Software (Thermo Fisher Scientific, Waltham, USA). The results were filtered on the basis of following criteria: (i) minimum and maximum fragment size obtained on agarose gel: $100 \mathrm{bp}$ and 2,000 bp, respectively (ii) minimum number of repeat units: 4.5. Twenty-four loci were then selected on the basis of $93 \%$ of conservation between the VNTRs.

The flanking sequences, of each VNTR locus, determined in the M. kansasii ATCC 12478 reference strain, and several other M. kansasii clinical strains ${ }^{65,66}$ with the CLC Genomics Workbench Software 8 (Qiagen, Nehren, Germany), were used to design oligonucleotide primers and PCR protocols with the

Vector NTI Software (Thermo Fisher Scientific, Waltham, USA) (Suppl. Table 2).

The PCR reactions were performed with a TopTaq Master Mix kit, as recommended by the manufacturer (Qiagen, Hilden, Germany) with $50 \mathrm{ng}$ of template DNA in a final volume of $25 \mu \mathrm{L}$. After initial denaturation at $95^{\circ} \mathrm{C}$ for $3 \mathrm{~min}$, the reaction mixture was run through 35 cycles of denaturation at $95^{\circ} \mathrm{C}$ for $30 \mathrm{~s}$, annealing at a temperature specific for particular VNTR loci (Suppl. Table 2) for $30 \mathrm{~s}$, and extension at $72{ }^{\circ} \mathrm{C}$ for $60 \mathrm{~s}$, followed by a final extension at $72{ }^{\circ} \mathrm{C}$ for $5 \mathrm{~min}$. The amplicons were separated electrophoretically at $3.5 \mathrm{~V} / \mathrm{cm}$ in $1 \%$ agarose gels in $0.5 \times$ TBE buffer and visualized by staining with ethidium bromide $(0.5 \mu \mathrm{g} / \mathrm{mL})$ and exposure to UV light $(\lambda=320 \mathrm{~nm})$.

Seven (i.e. VNTR 5, 9, 10, 12, 13, 16, 22) of the 24 selected loci were excluded from further analysis due to lack of amplification ${ }^{9,13,22}$ or multiple bands ${ }^{5,10,12,16,22}$, as seen upon electrophoresis.

To assign the number of alleles, at each VNTR locus, corresponding to the amplicon sizes, the amplicon-length-based allele calling table was used (Suppl. Table 3). The table was configured following the computer-assisted analysis of the VNTR sequences of the M. kansasii ATCC 12478 reference strain. Shortly, the in silico-deduced number of repeats, at each locus, was rounded to the closest integer value. The number of repeats, below or above this value were assigned to amplicons' lengths, calculated by adding or subtracting a multiple of the repeat size, at a given locus, from the amplicon size, determined for the M. kansasii ATCC 12478 reference strain.

For each isolate, the final result was a 17-digit VNTR code, corresponding to the number of repeats at each VNTR locus.

The VNTR copy number for loci containing 20 bp or less (i.e. VNTR 19, 20, 24) was verified for M. kansasii subtypes representatives by using Sanger technology (Suppl. Table 5). 
Cluster definition. A PFGE cluster was defined as two or more isolates assumed as clonal, based on the $80.4 \%$ cutoff value of the similarity between two PFGE patterns. A VNTR cluster was defined as two or more isolates sharing identical 17-loci VNTR typing profiles.

A PFGE-VNTR combined cluster was defined as two or more isolates sharing at least $80.4 \%$ cutoff value of the similarity between two PFGE patterns and identical 17-loci VNTR typing profiles.

Clustering rate was defined as percentage of clustered isolates among of all isolates genotyped ${ }^{67}$.

Construction of dendrograms. Similarities were calculated by the Cosine coefficient (PFGE and PFGE and VNTR typing combined) or Pearson's correlation coefficient (VNTR typing) algorithms. Dendrograms were constructed with BioNumerics ver. 5.0 software (Applied Maths, Sint-Martens-Latem, Belgium) by using the unweighted pair-group method with arithmetic averages (UPGMA) with $2 \%$ band position tolerance.

Calculation of discriminatory power. As a numerical index for the discriminatory power of each typing method, genetic diversity index (GDI) and Hunter and Gaston discriminatory index (HGDI) were used.

GDI was calculated, for each typing method, as a quotient of the total number of genetic patterns to the total number of isolates. The HGDI was calculated with the following formula:

$$
D I=1-\left[\frac{1}{N(N-1)}\right] \sum n j(n j-1),
$$

where $N$ is the total number of isolates, and $n j$ is the number of isolates representing each type ${ }^{68}$.

\section{References}

1. Onstad, G. D. Familial aggregations of group 1 atypical mycobacterial disease. Am. Rev. Resp. Dis 99, 426-429 (1969).

2. Ricketts, W. M., O’Shaughnessy, T. C. \& van Ingen, J. Human-to-human transmission of Mycobacterium kansasii or victims of a shared source? Eur. Respir. J. 44, 1085-1087 (2014).

3. Griffith, D. E. et al. ATS Mycobacterial Diseases Subcommittee; American Thoracic Society; Infectious Disease Society of America. An official ATS/IDSA statement: diagnosis, treatment, and prevention of nontuberculous mycobacterial diseases. Am. J. Respir. Crit. Care Med. 175, 367-416 (2007).

4. Hoefsloot, W. et al. The geographic diversity of nontuberculous mycobacteria isolated from pulmonary samples: A NTM-NET collaborative study. Eur. Respir. J. 42, 1604-1613 (2013).

5. Picardeau, M., Prod'hom, G., Raskine, L., LePennec, M. P. \& Vincent, V. Genotypic characterization of five subspecies of Mycobacterium kansasii. J. Clin. Microbiol. 35, 25-32 (1997).

6. Alcaide, F. et al. Heterogeneity and clonality among isolates of Mycobacterium kansasii: implications for epidemiological and pathological studies. J. Clin. Microbiol. 35, 1959-1964 (1997).

7. Taillard, C. et al. Clinical implications of Mycobacterium kansasii species heterogeneity: Swiss National Survey. J. Clin. Microbiol. 41, $1240-1244$ (2003).

8. Iwamoto, T. \& Saito, H. Comparative study of two typing methods $h s p 65$ PRA and ITS sequencing revealed a possible evolutionary link between Mycobacterium kansasii type I and II isolates. FEMS Microbiol. Let. 254, 129-133 (2005).

9. Iinuma, Y. et al. Large-restriction-fragment analysis of Mycobacterium kansasii genomic DNA and its application in molecular typing. J. Clin. Microbiol. 35, 596-599 (1997).

10. Gaafar, A. et al. Evaluation of a modified single-enzyme amplified-fragment length polymorphism technique for fingerprinting and differentiating of Mycobacterium kansasii type I isolates. J. Clin. Microbiol. 41, 3846-3850 (2003).

11. Chimara, E. C. et al. Molecular characterization of Mycobacterium kansasii isolates in the state of São Paulo between $1995-1998$. Mem. Inst. Oswaldo Cruz. 99, 739-743 (2004).

12. Santin, M. et al. Incidence and molecular typing of Mycobacterium kansasii in a defined geographical area in Catalonia, Spain. Epidemiol. Infect. 132, 425-432 (2004).

13. Zhang, Y. et al. Molecular analysis of Mycobacterium kansasii isolates from the United States. J. Clin. Microbiol. 42, 119-125 (2004).

14. da Silva Telles, M. A., Chimara, E., Ferrazoli, L. \& Riley, L. W. Mycobacterium kansasii: antibiotic susceptibility and PCR-restriction analysis of clinical isolates. J. Med. Microbiol. 54, 957-959 (2005).

15. Yoshida, S. et al. Molecular epidemiological analysis of Mycobacterium kansasii isolates. Kekkaku 82, 103-110 (2007).

16. Wu, T. S. et al. Clinical manifestations, antibiotic susceptibility and molecular analysis of Mycobacterium kansasii isolates from a university hospital in Taiwan. J. Antimicrob. Chemother. 64, 511-514 (2009).

17. Bakuła, Z. et al. Subtyping of Mycobacterium kansasii by PCR-Restriction Enzyme Analysis of the hsp65 gene. Biomed Res. Int. 1, 167954, https://doi.org/10.1155/2013/178725 (2013).

18. Thomson, R., Tolson, C., Huygens, F. \& Hargreaves, M. Strain variation amongst clinical and potable water isolates of $M$. kansasii using automated repetitive unit PCR. Int. J. Med. Microbiol. 304, 484-489 (2014).

19. Kwenda, G. et al. Molecular characterization of clinical and environmental isolates of Mycobacterium kansasii isolates from South African gold mines. J. Water Health 13, 190-202 (2015).

20. Li, Y. et al. Mycobacterium kansasii subtype I is associated with clarithromycin resistance in China. Front. Microbiol. 7, 2097 (2016).

21. Rónai, Z. et al. Molecular analysis and MIRU-VNTR typing of Mycobacterium avium subsp. paratuberculosis strains from various sources. J. Appl. Microbiol. 118, 275-283 (2015).

22. Bryant, J. M. et al. Whole-genome sequencing to identify transmission of Mycobacterium abscessus between patients with cystic fibrosis: a retrospective cohort study. Lancet. 381, 1551-1560 (2013).

23. Bryant, J. M. et al. Emergence and spread of a humantransmissible multidrug-resistant nontuberculous mycobacterium. Science 354, 751-757 (2016).

24. Supply, P., Magdalena, J., Himpens, S. \& Locht, C. Identification of novel intergenic repetitive units in a mycobacterial twocomponent system operon. Mol. Microbiol. 26, 991-1003 (1997).

25. Bull, T. J. et al. Mycobacterial interspersed repetitive units (MIRU) differentiate Mycobacterium avium subspecies paratuberculosis from other species of the Mycobacterium avium complex. Mol. Cell. Probes 17, 157-164 (2003).

26. Lavender, C. J. et al. Evaluation of VNTR typing for the identification of Mycobacterium ulcerans in environmental samples from Victoria, Australia. FEMS Microbiol. Lett. 287, 250-155 (2008).

27. Ichikawa, K. et al. Molecular typing of Mycobacterium intracellulare using multilocus variable-number of tandem-repeat analysis: identification of loci and analysis of clinical isolates. Microbiology 156, 496-504 (2010).

28. Sun, G., Chen, C. \& Li, J. Discriminatory potential of a novel set of variable number of tandem repeats for genotyping Mycobacterium marinum. Vet. Microbiol. 152, 200-204 (2011). 
29. Wong, Y. L., Ong, C. S. \& Ngeow, Y. F. Molecular Typing of Mycobacterium abscessus based on Tandem-Repeat polymorphism. J. Clin. Microbiol. 50, 3084-3088 (2012).

30. de Kruijf, M. et al. Low genetic diversity of bovine Mycobacterium avium subspecies paratuberculosis isolates detected by MIRUVNTR genotyping. Vet. Microbiol. 203, 280-285 (2017).

31. Eisenberg, T. et al. Phylogenetic and comparative genomics of the family Leptotrichiaceae and introduction of a novel fingerprinting MLVA for Streptobacillus moniliformis. BMC Genomics. 17, 864 (2016).

32. Yang, Y. et al. Genotyping Brucella canis isolates using a highly discriminatory multilocus variable-number tandem-repeat analysis (MLVA) assay. Sci. Rep. 7, 1067 (2017).

33. Jagielski, T. et al. Methodological and clinical aspects of the molecular epidemiology of Mycobacterium tuberculosis and other mycobacteria. Clin. Microbiol. Rev. 29, 239-290 (2016).

34. Radomski, N., Thibault, V. C. \& Karoui, C. Determination of genotypic diversity of Mycobacterium avium subspecies from human and animal origins by mycobacterial interspersed repetitive-unit-variable-number tandemrepeat and IS1311 restriction fragment length polymorphism typing methods. J. Clin. Microbiol. 48, 1026-1034 (2010).

35. Picardeau, M., Bull, T. J. \& Vincent, V. Identification and characterization of IS-like elements in Mycobacterium gordonae. FEMS Microbiol. Lett. 154, 95-102 (1997).

36. Zhou, K., Aertsen, A. \& Michiels, C. W. The role of variable DNA tandem repeats in bacterial adaptation. FEMS Microbiol. Rev. 38, 119-141 (2014)

37. Frothingham, R. \& Meeker-O'Connell, W. A. Genetic diversity in the Mycobacterium tuberculosis complex based on variable numbers of tandem DNA repeats. Microbiology 144, 1189-1196 (1998).

38. Hamblion, E. L. et al. Recent TB transmission, clustering and predictors of large clusters in London, 2010-2012: results from first 3 years of universal MIRU-VNTR strain typing. Thorax 71, 749-756 (2016).

39. Almaraz-Velasco, R. et al. A first insight into the genetic diversity of Mycobacterium tuberculosis in Veracruz, Mexico. Int. J. Mycobacteriol. 6, 14-20 (2017).

40. Vluggen, C. et al. Molecular epidemiology of Mycobacterium tuberculosis complex in Brussels, 2010-2013. PLoS One 12, e0172554, https://doi.org/10.1371/journal.pone.0172554 (2017).

41. Oelemann, M. C., Diel, R. \& Vatin, V. Assessment of an optimized mycobacterial interspersed repetitive-unit-variable-number tandem-repeat typing system combined with spoligotyping for population-based molecular epidemiology studies of tuberculosis. J. Clin. Microbiol. 45, 691-697 (2007).

42. Zheng, C. et al. Suitability of IS6110-RFLP and MIRU-VNTR for differentiating spoligotyped drug-resistant Mycobacterium tuberculosis clinical isolates from Sichuan in China. Biomed Res. Int. 1, 763204, https://doi.org/10.1155/2014/763204 (2014).

43. Ablordey, A. et al. Multilocus variable-number tandem repeat typing of Mycobacterium ulcerans. J. Clin. Microbiol. 43, 1546-1551 (2005).

44. Thibault, V. C. et al. New variable-number tandem-repeat markers for typing Mycobacterium avium subsp. paratuberculosis and $M$. avium strains: comparison with IS900 and IS1245 restriction fragment length polymorphism typing. J. Clin. Microbiol. 45, 2404-2410 (2007).

45. Dauchy, F. A. et al. Variable-number tandem-repeat markers for typing Mycobacterium intracellulare strains isolated in humans. BMC Microbiol. 29, 93 (2010).

46. Harris, K. A. et al. Molecular fingerprinting of Mycobacterium abscessus strains in a cohort of pediatric cystic fibrosis patients. J. Clin. Microbiol. 50, 1758-1761 (2012).

47. Hermans, P. W., van Soolingen, D. \& van Embden, J. D. A. Characterization of a major polymorphic tandem repeat in Mycobacterium tuberculosis and its potential use in the epidemiology of Mycobacterium kansasii and Mycobacterium gordonae. J. Bacteriol. 174, 4157-4165 (1992).

48. de Beer, J. L. et al. First worldwide proficiency study on variable-number tandem-repeat typing of Mycobacterium tuberculosis complex strains. J. Clin. Microbiol. 50, 662-669 (2012).

49. Yoshida, S. et al. Investigation of the population structure of Mycobacterium abscessus complex strains using 17-locus variable number tandem repeat typing and the further distinction of Mycobacterium massiliense hsp65 genotypes. J. Med. Microbiol. 64, 254-261 (2015).

50. Trovato, A. et al. Molecular typing of Mycobacterium abscessus isolated from cystic fibrosis patients. Int. J. Microbiol. 6, 138-141 (2017).

51. Inagaki, T. et al. Comparison of a variable-number tandem-repeat (VNTR) method for typing Mycobacterium avium with mycobacterial interspersed repetitive-unit-VNTR and IS1245 restriction fragment length polymorphism typing. J. Clin. Microbiol. 7,2156-2164 (2009).

52. Castellanos, E. et al. Molecular characterization of Mycobacterium avium subspecies paratuberculosis Types II and III isolates by a combination of MIRU-VNTR loci. Vet. Microbiol. 144, 118-126 (2010).

53. Rónai, Z., Csivincsik, Á., Dán, Á. \& Gyuranecz, M. Molecular analysis and MIRU-VNTR typing of Mycobacterium avium subsp. avium, 'hominissuis' and silvaticum strains of veterinary origin. Infect. Genet. Evol. 40, 192-199 (2016).

54. Jeon, S. et al. Molecular typing of Mycobacterium intracellulare using Pulsed-Field Gel Electrophoresis, Variable-Number TandemRepeat analysis, Mycobacteria Interspersed Repetitive-Unit-Variable-Number Tandem Repeat Typing, and Multilocus Sequence Typing: molecular characterization and comparison of each typing methods. Osong Public Health Res. Perspect. 5, 119-130 (2014).

55. Chen, K., Zhang, Y. \& Peng, Y. Variable-number tandem repeat markers for Mycobacterium intracellulare genotyping: comparison to the 16S rRNA gene sequencing. J. Infect. Dev. Ctries. 11, 158-165 (2017).

56. Iakhiaeva, E., McNulty, S. \& Brown Elliott, B. A. Mycobacterial interspersed repetitive-unit-variable-number tandem-repeat (MIRU-VNTR) genotyping of Mycobacterium intracellulare for strain comparison with establishment of a PCR-based database. J. Clin. Microbiol. 51, 409-416 (2013).

57. Merker, M. et al. The evolution of strain typing in the Mycobacterium tuberculosis complex. Strain variation in the Mycobacterium tuberculosis complex: its role in biology, epidemiology and control. Advances in Experimental Medicine and Biology (ed Gagneux, S.) 43-79 (Springer International Publishing, 2017).

58. van Ingen, J. \& van Soolingen, D. Molecular typing of nontuberculous mycobacteria. Molecular typing in bacterial infections. Infectious Disease (eds de Filippis, I. \& McKee, M. L.) 167-177 (Humana Press, 2013).

59. Sampaio, J. L. et al. Application of four molecular typing methods for analysis of Mycobacterium fortuitum group strains causing post-mammaplasty infections. Clin. Microbiol. Infect. 12, 142-149 (2006).

60. Butler, W. R. et al. Standardized method for HPLC identification of mycobacteria. HPLC users group in cooperation with Centers for Disease Control and Prevention, Atlanta: U.S. Public Health Service, CDC. (1996).

61. Telenti, A. et al. Rapid identification of mycobacteria to the species level by polymerase chain reaction and restriction enzyme analysis. J. Clin. Microbiol. 31, 175-178 (1993).

62. Kim, B. J. et al. Differentiation of mycobacterial species by PCR-restriction analysis of DNA (343 base pairs) of the RNA polymerase gene $(r p o B)$. J. Clin. Microbiol. 39, 2102-2109 (2001).

63. Bakuła, Z. et al. Proposal of a new method for subtyping of Mycobacterium kansasii based upon PCR restriction enzyme analysis of the tuf gene. Diagn. Microbiol. Infect. Dis. 84, 318-321 (2016).

64. Benson, G. Tandem repeats finder: a program to analyze DNA sequences. Nucleic Acids Res. 27, 573-580 (1999). 
65. Strapagiel, D. et al. Draft genome sequences of Mycobacterium kansasii strains 1010001454, 1010001458, 1010001468, 1010001493, 1010001495, and 1010001469, isolated from environmental sources. Genome Announc. 2, 3 (2016).

66. Borówka, P. et al. Draft genome sequences of Mycobacterium kansasii clinical strains. Genome Announc. 5, 22 (2017).

67. Durmaz, R. et al. Population-based molecular epidemiological study of tuberculosis in Malatya, Turkey. J. Clin. Microbiol. 45, 4027-35 (2007).

68. Hunter, P. R. \& Gaston, M. A. Numerical index of the discriminatory ability of typing systems: an application of Simpson's index of diversity. J. Clin. Microbiol. 26, 2465-6 (1988).

\section{Acknowledgements}

The study was financed by the National Centre for Research and Development «LIDER» Programme [LIDER/044/457/L-4/12/NCBR/2013]. We would like to thank Dr. Vit Ulmann, Institute of Public Health, Ostrava, Czech Republic and Dr. Miren Josebe Unzaga-Baranano, Clinical Microbiology and Infection Control, Basurto Hospital, Spain for kindly donating the strains for this study. We would also like to thank Dr. Arkadiusz Wojtasik, Proteon Pharmaceuticals S.A., Łódż, Poland, for his assistance in genotyping data analysis.

\section{Author Contributions}

Z.B.: data acquisition (PFGE and VNTR typing performance), analysis and interpretation, manuscript writing; A.B., A.Z., I.S.-K.: data acquisition (VNTR typing performance); P.B.: computer-assisted analysis of genotyping data; A.P.: data acquisition (VNTR typing performance); D.S.: VNTR in silico flanking analysis; P.P.: genome based in silico determination of VNTRs; J.D.: VNTR primers design, supervision of VNTR typing, critical revision; J.I.: critical revision; M.P.: data acquisition (strain initial typing); J.B.: critical revision; T.J.: study design and supervision, manuscript drafting, critical revision.

\section{Additional Information}

Supplementary information accompanies this paper at https://doi.org/10.1038/s41598-018-21562-z.

Competing Interests: The authors declare no competing interests.

Publisher's note: Springer Nature remains neutral with regard to jurisdictional claims in published maps and institutional affiliations.

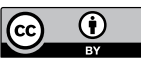

Open Access This article is licensed under a Creative Commons Attribution 4.0 International License, which permits use, sharing, adaptation, distribution and reproduction in any medium or format, as long as you give appropriate credit to the original author(s) and the source, provide a link to the Creative Commons license, and indicate if changes were made. The images or other third party material in this article are included in the article's Creative Commons license, unless indicated otherwise in a credit line to the material. If material is not included in the article's Creative Commons license and your intended use is not permitted by statutory regulation or exceeds the permitted use, you will need to obtain permission directly from the copyright holder. To view a copy of this license, visit http://creativecommons.org/licenses/by/4.0/.

(c) The Author(s) 2018 